\title{
Impaired Interhemispheric Synchrony in Bronchial Asthma
}

\author{
Ya-Jun $\mathrm{Wu}\left(\mathbb{D}^{1}{ }^{1, *}\right.$ \\ Jie Raol,* \\ Xin Huang ${ }^{2}$ \\ $\mathrm{Na} \mathrm{Wu}{ }^{\prime}$ \\ Ling Shi' \\ Hui Huang' \\ $\mathrm{Si}-\mathrm{Yu} \mathrm{Li}{ }^{1}$ \\ Xiao-Lin Chen' \\ Shui-Qin Huang' \\ Pei-Pei Zhong' \\ Xiao-Rong Wu (D' \\ Jun Wang $\mathbb{D}^{3}$
}

'Department of Ophthalmology, The First Affiliated Hospital of Nanchang University, Nanchang, Jiangxi Province, People's Republic of China; ${ }^{2}$ Department of Ophthalmology, Jiangxi Provincial People's Hospital Affiliated to Nanchang University, Nanchang, Jiangxi Province, People's Republic of China; ${ }^{3}$ Department of Respiratory Disease, Jiangxi Provincial People's Hospital Affiliated to Nanchang University, Nanchang, Jiangxi Province,

People's Republic of China

*These authors contributed equally to this work
Correspondence: Jun Wang

Email wangjun5087@163.com
Purpose: Converging evidence demonstrated that bronchial asthma (BA) individuals with hypoxia were associated with functional and morphological reorganization in the brain. However, the alterations of the interhemispheric functional connectivity in BA individuals remain unknown. The purpose of this study was to assess the interhemispheric functional connectivity changes in individuals with hypoxia due to middle-aged BA using voxelmirrored homotopic connectivity (VMHC) methods.

Methods: In total, $31 \mathrm{BA}$ individuals and 30 healthy controls (HCs) closely matched in age, sex, and education underwent resting-state magnetic resonance imaging (MRI) scans. VMHC analysis was performed to investigate differences in interhemispheric functional connectivity between the two groups. Then, a seed-based resting-state functional connectivity (rsFC) analysis was conducted to further reveal the abnormal functional connectivity between the altered VMHC regions and the whole brain.

Results: Compared with HCs, BA individuals had significantly lower VMHC values in the bilateral basal ganglia/thalamus/insula, cuneus/calcarine/lingual gyrus, precentral and postcentral gyrus. [voxel level $\mathrm{P}<0.01$, Gaussian random field (GRF) correction, cluster level $\mathrm{P}<0.05]$. Taking VMHC altered brain areas as seed points, the rsFC values of left insula/ supramarginal/postcentral gyrus (PostCG)/inferior parietal lobule (IPL) brain areas in BA were increased.

Conclusion: The abnormal resting-state functional connectivity of middle-aged BA is altered in specific brain regions related to the basal ganglia network, visual network, and sensorimotor network, which may be related to the neuropathogenesis of asthma patients. Furthermore, these VMHC and FC values may be important clinical indicators for the diagnosis and treatment of asthma patients.

Keywords: bronchial asthma, voxel-mirrored homotopic connectivity, functional magnetic resonance imaging, basal ganglia network, visual network, sensorimotor network

\section{Introduction}

Asthma is a global health problem; nearly 300 million people suffer from asthma globally. ${ }^{1}$ According to a recent survey in China, the prevalence of asthma is $1.24 \% .^{2}$ Risk factors include but are not limited to obesity, respiratory infections, ${ }^{3}$ genetic, ${ }^{4}$ and environmental factors. ${ }^{5}$ Chronic inflammation of the airways is the main pathological mechanism of asthma, subsequently resulting in narrowing of the airway and the classic symptoms of wheezing. Bronchial asthma (BA) is a chronic respiratory disease characterized by chronic airway inflammation and bronchial hyper reaction, affecting all age groups from infants to the elderly. During the attack, patients mainly present with wheezing, shortness of breath, and dyspnea, resulting in impaired lung function ${ }^{6}$ and hypoxia. However, BA patients are not 
only associated with respiratory symptoms but also suffer from mental problems. At present, BA individuals have been shown to face higher risks of occurrence of depression and anxiety than healthy individuals. ${ }^{7-9}$ In addition, studies have shown that the incidence of cognitive impairment in BA individuals is higher than that in healthy individuals. ${ }^{10}$ Thus, BA patients might lead to the dysfunction of the central nervous system.

To date, accumulated neuroimaging researches demonstrated that BA leads to significant brain functional and structural architecture changes. Xiong et al reported that the regional homogeneity of the right insula (ReHo) decreased in asthma patients without depression, while the regional homogeneity of the right insula (ReHo) decreased in asthma patients with depression. ${ }^{11}$ Kline et al found that the insula and anterior cingulate cortex might play a critical role in inflammatory processes in BA. ${ }^{12,13}$ Meanwhile, the BA patients showed increased functional connectivity between the left ventral anterior insula and the right anterior cingulate cortex (ACC), decreased functional connectivity between the left ventral anterior insula and the bilateral parietal lobe relative to HCs. ${ }^{14}$ Moreover, the asthma patients showed abnormal functional network centrality in the default mode network, the frontoparietal network, and the sensorimotor network. ${ }^{15}$ In addition, BA leads to specific brain structural changes. Carlson et al revealed that smaller hippocampal volume was detected in asthma patients, which might have implications for impaired memory in BA individuals. ${ }^{16}$ Voxel-based morphometry (VBM) study reported that gray matter (GM) volumes of the insular cortex and the brainstem periaqueductal grey were observed and it correlated with ratings of dyspnea unpleasantness in asthma patients. ${ }^{17}$ Bian et al reported that the asthma patients had abnormal white matter (WM) integrity in the left forceps major, cingulum, and right uncinate fasciculus, inferior longitudinal fasciculus relative to healthy individuals. ${ }^{18}$ However, the abovementioned studies focused on the specific brain region functional and structural changes. The alternations of interhemispheric connectivity occurring in BA patients remain unknown. We hypothesized that BA might be associated with impaired interhemispheric connectivity changes implicated for impaired cognition and depression. Also, we want to evaluate the abnormal state of the same brain area in both cerebral hemispheres.

The human brain consists of two hemispheres with extensive anatomical and functional connections between homologous sites. The synchrony of homotopic connectivity is an important feature of the functional structure of the brain. Interhemispheric coordination is involved in important physiological functions. Previous electroencephalogram (EEG) studies demonstrated that interhemispheric coupling was closely contributed to the processing of motor, auditory, and vision functions. ${ }^{19-21}$ Voxel-mirrored homotopic connectivity (VMHC) is an effective Restingstate-fMRI approach that can be used to investigate the homotopic connectivity between each hemisphere. ${ }^{22}$ VMHC method has been shown high test-retest reliability. The VMHC method had been successful to assess the neurophysiological mechanism of many diseases such as Obstructive Sleep Apnea-Hypopnea Syndrome ${ }^{23}$ and chronic insomnia disorder. ${ }^{24}$ However, the long-term effect of intermittent dyspnea due to BA on homotopic connectivity is not well understood.

Here, the purpose of the study was to explore the interhemispheric connectivity changes in middle-aged BA individuals. Furthermore, a seed-based resting-state functional connectivity (rsFC) analysis was applied to investigate the functional networks architecture seed as the altered VMHC regions in BA individuals. The relationship between the VMHC index and clinical variables was analyzed by Pearson correlation. These findings might offer important insights into the understanding of abnormal hemispheric communication with BA individuals.

\section{Materials and Methods}

\section{Participants}

A total of 31 patients with BA (17 men and 14 women) were admitted to the Jiangxi Provincial People's Hospital (Nanchang, Jiangxi Province, China). The diagnostic criteria for bronchial asthma were as follows: (1) presence of intermittent hypoxia and wheezing symptoms; (2) more than $20 \%$ decrease in the rate of forced expiratory volume within $1 \mathrm{~h}$; (3) absence of an upper respiratory tract infection; and (4) absence of psychiatric and cerebrovascular disorders.

Thirty HCs (15 men, 15 women) age, sex, and education status matched with patients in the BA group were enrolled. All healthy participants fulfilled the following criteria: (a) no psychiatric disorders (eg, depression, bipolar disorder, sleep disorder, etc.); (b) without any contraindications of MRI; (c) no metal foreign bodies in or on the body surface of the subjects. All research methods were approved by the Ethics Committee of People's 
Hospital affiliated Nanchang University. Informed consent was obtained from all participants before MRI scanning, and the research methods complied with the principles of the Declaration of Helsinki. Please refer to the Supplementary Materials for MRI parameters and fMRI data preprocessing parts. $^{25-27}$

\section{Voxel-Mirrored Homotopic Connectivity Analysis}

According to a previous study, ${ }^{22}$ the VMHC was calculated with REST software (http://www.resting-fmri.source forge.net). ${ }^{28}$ Briefly, the homotopic FC for each subject was computed as the Pearson correlation coefficient between each voxel's residual time series and that of its symmetrical interhemispheric counterpart. Correlation values were then Fisher $z$-transformed to improve normality.

\section{Seed-Based Resting State Functional Connectivity (rsFC) Analysis}

To further detect the altered functional networks behind the impaired $\mathrm{VMHC}$, the brain regions of decreased VMHC values were selected as the regions of interest (ROIs). Correlation analysis of the time course was performed between the spherical seed region and each voxel of the whole brain for each subject using REST software. Then, all functional connectivity maps were z-transformed with Fisher's r-to-z transformation to reduce the influence of individual variation for group statistical comparisons.

\section{Statistical Analysis}

A one-sample-test was conducted to assess intra-group patterns of z-VMHC maps using SPM8 software. Twosample t-tests including general linear model (GLM) for the groups of VMHC using SPM 8 (with age and gender treated as covariates; voxel-wise $\mathrm{P}<0.01$, Gaussian random field (GRF) theory connected cluster level, $\mathrm{P}<0.05)$.
A two-sample $t$-test was used to compare two group differences in the zFC maps including GLM for the groups of VMHC using SPM 8 (with age and gender treated as covariates; voxel-wise $\mathrm{P}<0.01$, Gaussian random field (GRF) theory connected cluster level, $\mathrm{P}<0.05$ ).

Besides, a one-sample $t$-test was used to assess the positively correlated regions. We only focus on the positive correlation results of $\mathrm{FC}$, because $\mathrm{FC}$ with negative correlation is controversial at present. We analyzed the differences between $\mathrm{BA}$ and $\mathrm{HC}$ in the positive connectivity regions of seed by GLM (voxel-wise $\mathrm{P}<0.01$, GRF theory connected cluster level, $\mathrm{P}<0.05)$.

The receiver operator characteristic (ROC) curve was used to assess the sensitivity of the $\mathrm{z}-\mathrm{VMHC}$ values to distinguish the $\mathrm{BA}$ and $\mathrm{HC}$ groups. Pearson correlation was performed to investigate the relationship between the $\mathrm{z}-\mathrm{VMHC}$ and $\mathrm{zFC}$ values and clinical variables in BA groups.

\section{Results}

\section{Demographic and Clinical Characteristics of Subjects}

Thirty-one patients with BA (seventeen males and fourteen females, mean age: $51.62 \pm 5.20$ years) and $30 \mathrm{HCs}$ (seventeen males and fourteen females, mean age: 51.26 \pm 5.27 years) were included in this study. There were no significant differences in gender, age, weight, and handedness between $\mathrm{BA}$ and $\mathrm{HC}$ groups $(P>0.05)$. Details are presented in Table 1.

\section{The Difference of VMHC Between the BA Group and HC Group}

The spatial pattern of VMHC at the average level of BA and $\mathrm{HC}$ groups is shown as in Figure 1 (voxel-level $\mathrm{P}<0.01$, GRF-correction, cluster level $\mathrm{P}<0.05$ ). (Because the VMHC results of the LH and the RH of each group are the same, we took the LH of the BA group and the RH of the

Table I Demographics and Clinical Measurements Between BA and HC Groups

\begin{tabular}{|l|l|l|l|l|}
\hline Condition & BA & HC & t & P-value* \\
\hline Male/female & $17 / 14$ & $15 / 15$ & N/A & 0.705 \\
Age (years) & $51.62 \pm 5.20$ & $51.26 \pm 5.27$ & 0.260 & 0.796 \\
Weight (kg) & $63.56 \pm 7.12$ & $63.18 \pm 6.68$ & 0.197 & 0.845 \\
Handedness & $20 \mathrm{R}$ & $20 \mathrm{R}$ & N/A & $>0.99$ \\
Duration of Asthma (years) & $27 \pm 6.21$ & N/A & N/A & N/A \\
\hline
\end{tabular}

Notes: *P $<0.05$ Independent t-tests comparing two groups, the data was showed in mean \pm standard deviation. Abbreviations: HC, healthy control; N/A, not applicable; 


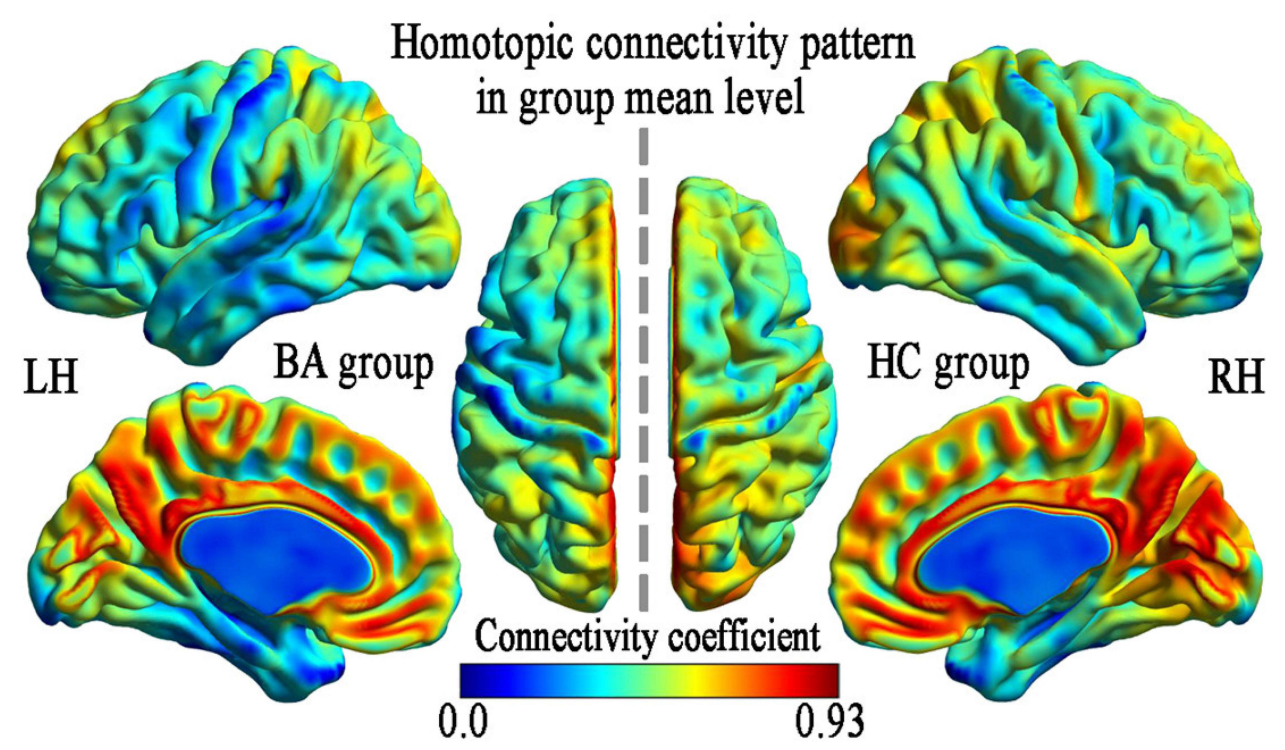

Figure I VMHC spatial patterns at the group mean level of the BA and $\mathrm{HC}$ groups.

Note: Within-group mean VMHC maps within the BA (left) and HCs (right).

Abbreviations: VMHC, voxel-mirrored homotopic connectivity; BA, bronchial asthma; HC, health control; $\mathrm{LH}$, left hemisphere; $\mathrm{RH}$, right hemisphere.

HC group respectively for the beauty of the resulting map.) The VMHC value of BA in bilateral ganglia/thalamus/ Insula, cuneus/Calcarine/lingual gyrus, precentral gyrus (PreCG), and postcentral gyrus (PostCG) were lower than that of HCs. (voxel-level P $<0.01$, GRF-correction, cluster level $\mathrm{P}<0.05$ ) Details are presented in Table 2 and Figure 2.

\section{Comparative Analysis of the Functional Connectivity of VMHC Altered Brain Regions in BA Group and HC Group}

The altered VMHC of each brain region was used as the seed brain region, compared with the control group, the rsFC values of the left insula/supramarginal/PreCG/inferior parietal lobule (IPL) brain areas in the BA group were significantly increased in those of control group, while the rsFC values in the right basal ganglia/thalamus, left caudate/ anterior cingulate and bilateral cuneus/calcarine/lingual gyrus/PreCG/PostCG were significantly decreased in BA group. (voxel-level $\mathrm{P}<0.01$, GRF-correction, cluster level $\mathrm{P}<0.05)$ Details are presented in Table 3 and Figures 3-5.

\section{Receiver Operating Characteristic (ROC) Curve Analysis}

The receiver operating Characteristic curve (ROC) tested the different combinations of abnormal mean VMHC values of 8 bilateral brain regions in the two groups, and we used the ROC curve to analyze the diagnostic value of different brain regions. The areas under the curve (AUC) were as follows: bilateral-basal ganglia(BG)/thalamus (Tha)/insula (Ins) was 0.943; bilateral-cuneus (Cun)/ calcarine (Cal)/lingual Gyrus(LG) was 0.852 ; bilateralprecentral Gyrus (M1)/postcentral Gyrus (S1) was 0.867,

Table 2 Differences in VMHC Between Two Groups

\begin{tabular}{|l|l|l|l|l|l|l|}
\hline Brain Region & \multirow{2}{*}{ BA } & Voxels Size & Peak t-value & \multicolumn{3}{|l|}{ Peak Coordinates in MNI Space } \\
\cline { 4 - 6 } & & & & $\mathbf{X}$ & $\mathbf{Y}$ \\
\hline BA <HC & & & & & \\
Basal ganglia/Thalamus/ Insula & - & 557 & -6.591 & \pm 42 & -06 & 15 \\
Cuneus/ Calcarine/ Lingual Gyrus & $17,18,19$ & 359 & -6.423 & \pm 09 & -93 & 0 \\
PreCG /PostCG & $3,4,6$ & 211 & -6.250 & \pm 66 & 0 \\
\hline
\end{tabular}

Notes: t: statistical value of peak voxels showing significant differences between the two groups. $\mathrm{X}, \mathrm{Y}, \mathrm{Z}$ are the coordinates of primary peak locations in the MNI space. (voxel level $P<0.01$, GRF-corrected for multiple comparisons at a cluster level of $P<0.05$ ).

Abbreviations: VMHC, voxel-mirrored homotopic connectivity; GRF, Gaussian random field; BA, bronchial asthma; HCs, healthy controls; MNI, Montreal Neurological Institute; BA's, Brodmann's area; PreCG, precentral Gyrus; PostCG, postcentral gyrus. 


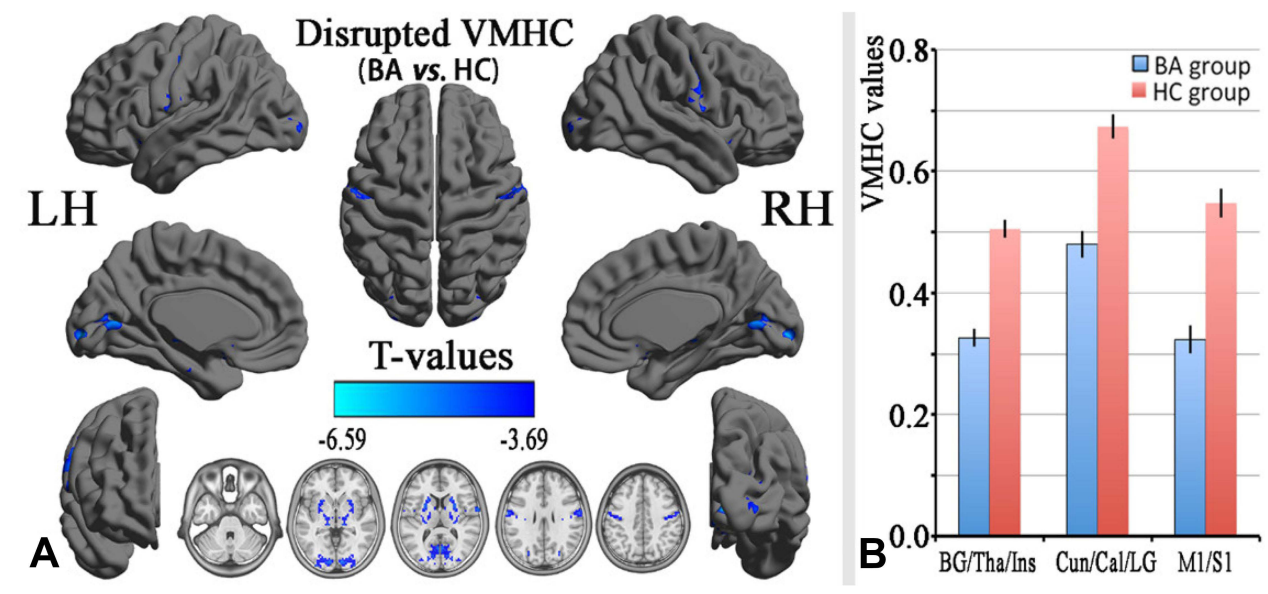

Figure 2 Group comparisons of the VMHC between the BA and HCs.

Notes: Significant VMHC differences were found between two groups. (A) The blue areas indicate lower VMHC values, respectively (two-tailed, voxel-level P < 0.0I, GRF correction, cluster-level $\mathrm{P}<0.05$ ). The mean values of altered VMHC values between the BA and $\mathrm{HC}$ groups were shown in the histogram. (B)

Abbreviations: VMHC, voxel-mirrored homotopic connectivity; BA, bronchial asthma; HC, health control; LH, left hemisphere; RH, right hemisphere; BG, basal ganglia; Tha, thalamus; Ins, insula; Cun, cuneus; Cal, calcarine; LG, lingual gyrus; MI, precentral gyrus; SI, postcentral gyrus.

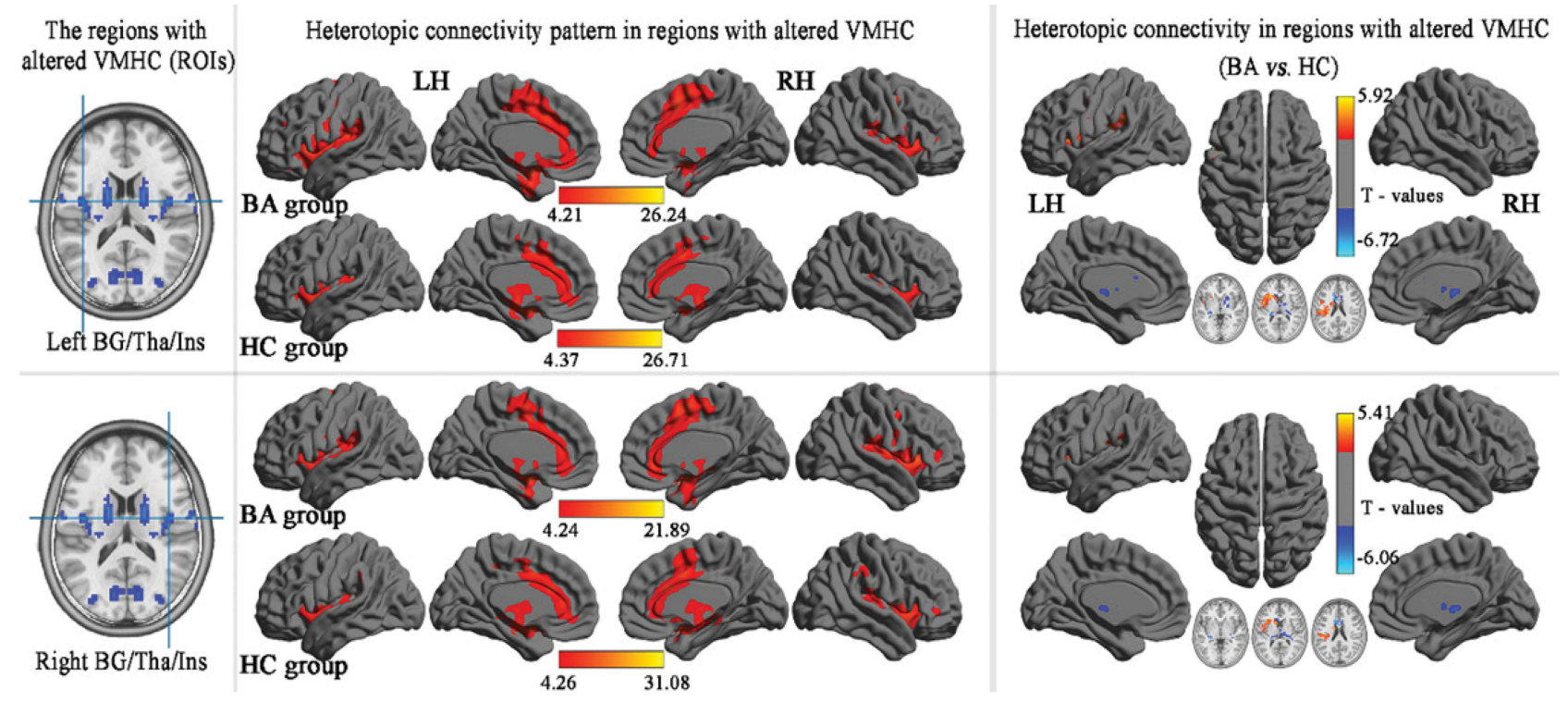

Figure 3 Group comparisons seed-based FC of the altered VMHC between the BA and HC seeded regions in bilateral BG/Tha/Ins. (P $<0.0 \mathrm{I}$, GRF-corrected at a cluster level of $P<0.05)$.

Abbreviations: VMHC, voxel-mirrored homotopic connectivity; GRF, Gaussian random field; BA's, Brodmann's area; BA, bronchial asthma; HCs, healthy controls; FC, functional connectivity; ROI, region of interest; BG, basal ganglia; Tha, thalamus; Ins, insula.

B-BG/Tha/Ins showed the highest AUG, which may mean that these brain regions are most important for the diagnosis of BA. Details are presented in Figure 6.

\section{Correlation Analysis}

At the level of GRF correction $\mathrm{P}<0.05$, VMHC anomalies of BA were not correlated with clinical variables (the duration, age, gender, and bodyweight of BA patients.).

\section{Discussion}

In this study, rsMRI technology was used to detect middleaged BA and HC controls, and the VMHC method was used to evaluate the changes in the functional connections between cerebral hemispheres. We have two major findings, first, it was mainly found that the VMHC values of these brain areas (bilateral basal ganglia/thalamus/insula, cuneus/ calcarine/lingual gyrus, PreCG, and PostCG) in 


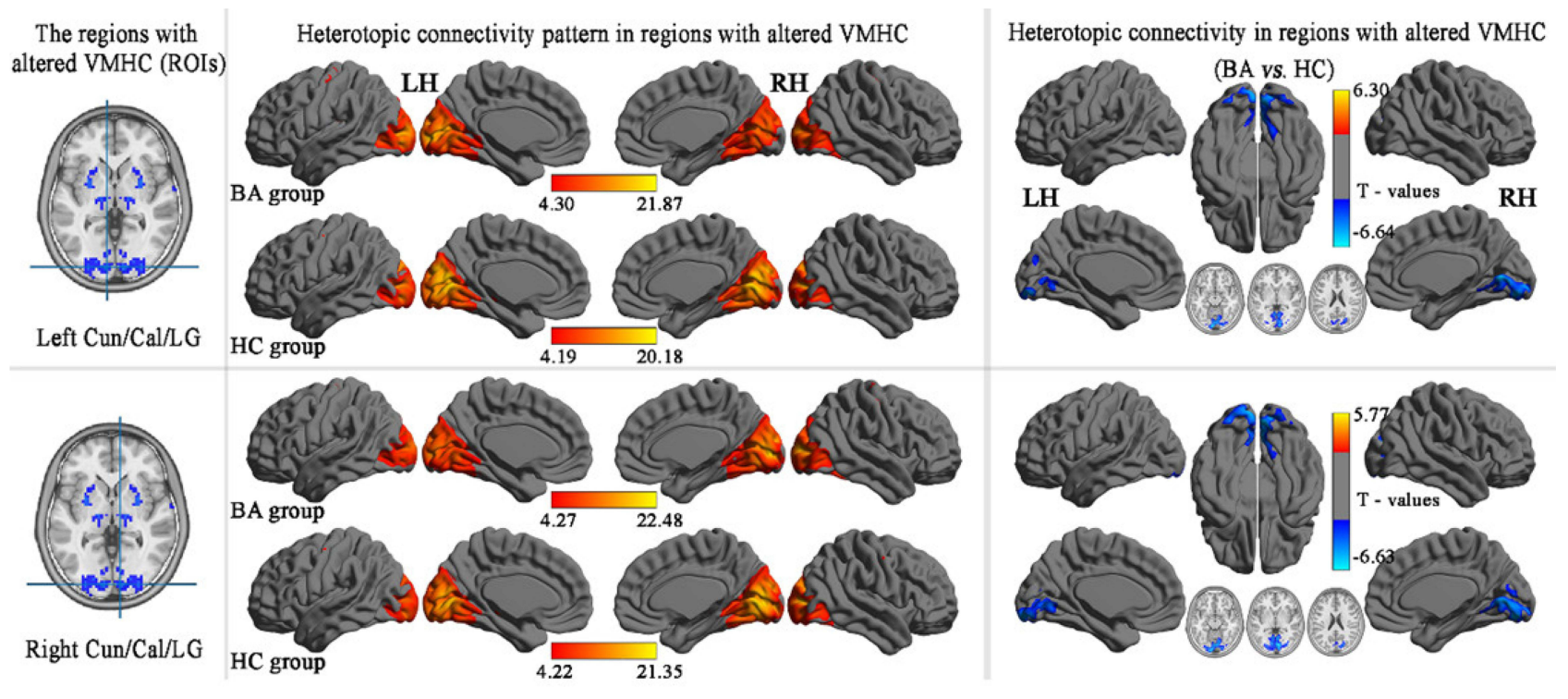

Figure 4 Group comparisons seed-based FC of the altered VMHC between the BA and HC seed regions in bilateral Cun/Cal/LG. (P $<0.0 \mathrm{I}$, GRF-corrected at a cluster level of $P<0.05$ ).

Abbreviations: VMHC, voxel-mirrored homotopic connectivity; GRF, Gaussian random field; BA's, Brodmann's area; BA, bronchial asthma; HCs, healthy controls; FC, functional connectivity; ROI, region of interest; Cun, cuneus; Cal, calcarine; LG, lingual gyrus.

the BA were significantly lower than those in the normal controls. Second, functional connectivity was studied using VMHC abnormal brain regions as seed points, compared with the HC group. As far as we know, this is the first report that VMHC combined with seed-based rsFC method which has been used to study the changes of functional connectivity in the whole brain of BA. We believe that these findings will contribute to the

Table 3 Differences in Heterotopic Connectivity of the Regions with Altered VMHC

\begin{tabular}{|c|c|c|c|c|c|c|}
\hline \multirow[t]{2}{*}{ Brain Region } & \multirow[t]{2}{*}{ BA } & \multirow[t]{2}{*}{ Voxels Size } & \multirow[t]{2}{*}{ Peak t-value } & \multicolumn{3}{|c|}{ Peak Coordinates in MNI Space } \\
\hline & & & & $\mathbf{x}$ & $\mathbf{Y}$ & $\mathbf{Z}$ \\
\hline ROII: L-Basal ganglia/Thalamus/ Insula & & & & & & \\
\hline L- Insula/ SupraMarginal/ PreCG /IPL & $13,6,40$ & 1082 & 5.923 & -42 & -39 & 24 \\
\hline R- Basal ganglia/Thalamus & - & 356 & -6.269 & -27 & -30 & 3 \\
\hline ROI2: R-Basal ganglia/Thalamus/ Insula & & & & & & \\
\hline R- Basal ganglia/Thalamus & - & 271 & -5.474 & -24 & -30 & 6 \\
\hline L- Insula/ SupraMarginal/ PreCG /IPL & - & 293 & 4.801 & -27 & 24 & 9 \\
\hline L-Caudate/Anterior Cingulate & 33 & 229 & -6.059 & -12 & 12 & 27 \\
\hline ROI3: L- Cuneus/ Calcarine/ Lingual Gyrus & & & & & & \\
\hline $\begin{array}{l}\text { B-Cuneus/ Calcarine/ Lingual Gyrus } \\
\text { ROI4: R-Cuneus/ Calcarine/ Lingual Gyrus }\end{array}$ & $17,18,19$ & 1506 & -6.644 & 3 & -69 & 6 \\
\hline $\begin{array}{l}\text { B- Cuneus/ Calcarine/ Lingual Gyrus } \\
\text { ROI5: L-PreCG/PostCG }\end{array}$ & $17,18,19$ & 1863 & -6.628 & 15 & -93 & -9 \\
\hline R- PreCG/PostCG & $3,4,6$ & 490 & -6.136 & 39 & -18 & 33 \\
\hline L-PreCG/PostCG & $3,4,6$ & 275 & -5.486 & -42 & -21 & 30 \\
\hline ROI6: R- PreCG/PostCG & & & & & & \\
\hline R- PreCG/PostCG & $3,4,6$ & 358 & -6.104 & 42 & -18 & 33 \\
\hline L-PreCG/PostCG & $3,4,6$ & 105 & -5.114 & -42 & -21 & 30 \\
\hline
\end{tabular}

Notes: t: statistical value of peak voxels showing significant differences between the two groups (negative values: $\mathrm{BA}<\mathrm{HC}$; positive values: $\mathrm{BA}>\mathrm{HC}$ ). $\mathrm{X}$, $\mathrm{Y}, \mathrm{Z}$ are the coordinates of primary peak locations in the MNI space. (voxel level $\mathrm{P}<0.0 \mathrm{I}$, GRF-corrected for multiple comparisons at a cluster level of $\mathrm{P}<0.05$ ).

Abbreviations: VMHC, voxel-mirrored homotopic connectivity; GRF, Gaussian random field; BA's, Brodmann's area; MNI, Montreal Neurological Institute Coordinate System; BA, bronchial asthma; HCs, healthy controls; MNI, Montreal Neurological Institute; BA's, Brodmann's area; IPL, inferior parietal lobule; PreCG, precentral gyrus; PostCG, postcentral gyrus; L, left; R, right; B, bilateral. 


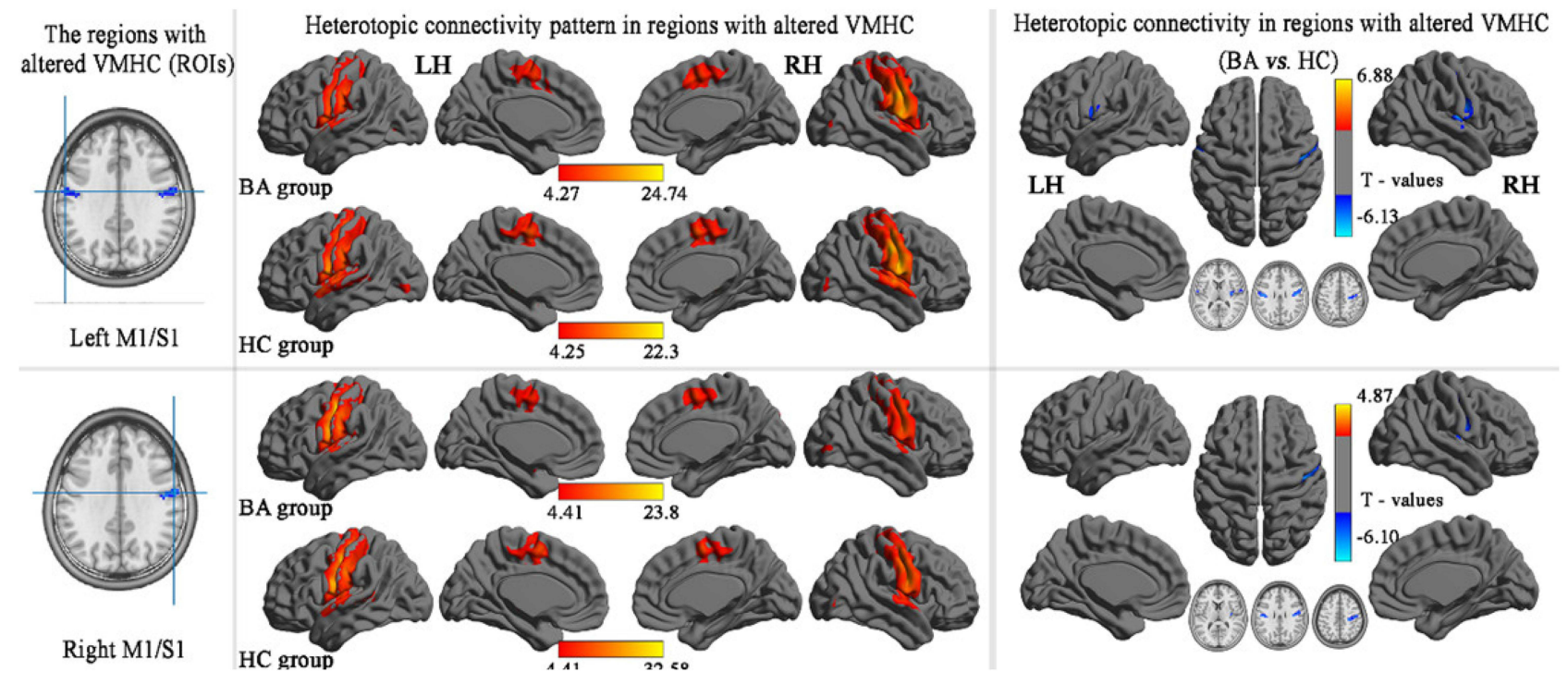

Figure 5 Group comparisons seed-based FC of the altered VMHC between the BA and $\mathrm{HC}$ seed regions in bilateral MI/SI. (P $<0.0 \mathrm{I}, \mathrm{GRF}$-corrected at a cluster level of $P<0.05)$.

Abbreviations: VMHC, voxel-mirrored homotopic connectivity; GRF, Gaussian random field; BA's, Brodmann's area; BA, bronchial asthma; HCs, healthy controls; FC, functional connectivity; ROI, region of interest; MI, precentral Gyrus; SI, postcentral gyrus.

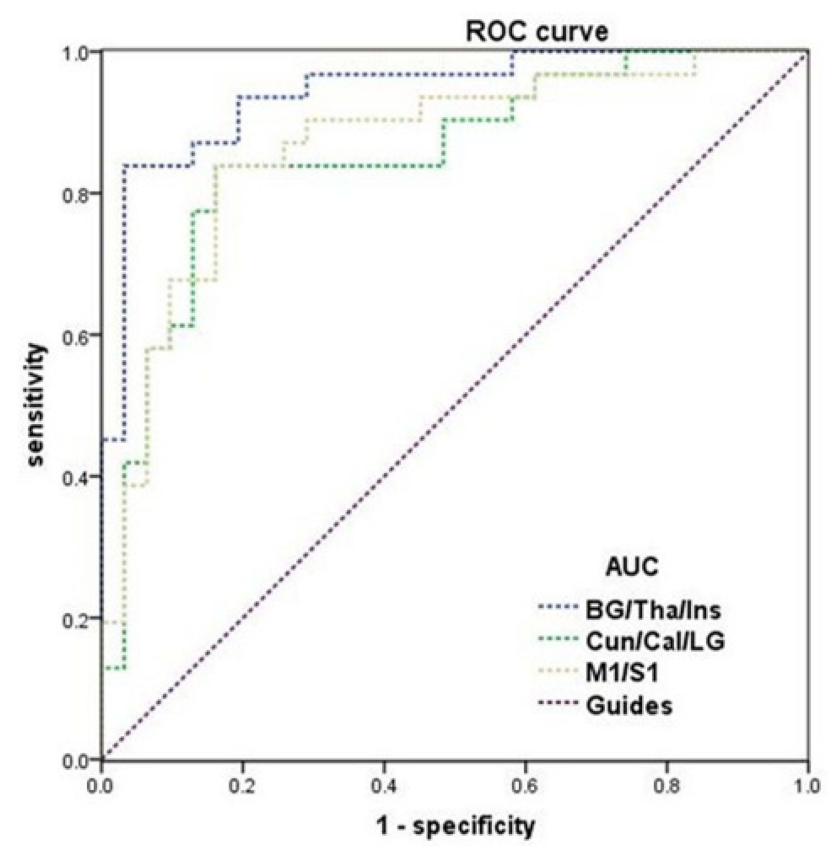

Figure 6 High sensitivity in mean VMHC values of different brain regions within two groups.

Notes: ROC curve in VMHC: BA $<$ HC, for B-BG/Tha/Ins $0.943(\mathrm{P}<0.001$; $95 \% \mathrm{Cl}$ : 0.887-0.999); BA< HC, for B-Cun/Cal/LG 0.852 (P<0.00I; 95\% Cl: 0.755-0.950); $\mathrm{BA}<\mathrm{HC}$, for $\mathrm{B}-\mathrm{MI} / \mathrm{SI} 0.867$ ( $\mathrm{P}<0.00 \mathrm{I} ; 95 \% \mathrm{Cl}: 0.774-0.960)$;

Abbreviations: ROC, receiver operating characteristic; AUC, area under the curve; VMHC, voxel-mirrored homotopic connectivity; BA, bronchial asthma; $\mathrm{HC}$, health control; BG, basal ganglia; Tha, thalamus; Ins, insula; Cun, cuneus; Cal, calcarine; LG, lingual gyrus; MI, precentral gyrus; SI, postcentral gyrus; B, bilateral.

development of an imaging biomarker for the diagnosis of $\mathrm{BA}$, which is of great significance in improving the clinical efficacy and quality of life of patients with asthma.
Functional homotopy refers to the high similarity in endogenous spontaneous activities of neurons of the same origin in the left and right hemispheres of the brain, and it is related to brain stratification. ${ }^{22}$ The VMHC method measures interhemispheric coordination and is a rsMRI method used to study the functional connections between hemispheres; also, it is an important method to study brain information integration by measuring the correlation between cerebral hemisphere blood oxygen leveldependent time series and reflecting the information exchange and integration mode between cerebral hemispheres. ${ }^{29}$ At present, this method has been widely used to assess the functional connectivity of the cerebral hemisphere in various neuropsychiatric diseases. Meanwhile, asthma is considered to be a respiratory disease that seriously affects the central nervous system. ${ }^{30}$ Combined with our study, the VMHC values of eight brain regions, namely bilateral basal ganglia/thalamus/insula, cuneus/calcarine/lingual gyrus, PreCG, and PostCG decreased in BA patients, suggesting that the functional connectivity in these brain regions is impaired in BA patients.

The basal ganglia network plays an important role in emotion, association, and limbic processing, which is mainly responsible for cognition, motor, and emotion change. ${ }^{31}$ Thalamus and basal ganglia are important parts of the basal ganglia network, ${ }^{32}$ the abnormal brain area of 
the network is likely to cause damage to the corresponding cognitive, motor, or other functions. Xiaodan et al found that BOLD signal activation in the middle gyrus, occipital gyrus, lingual gyrus, and thalamus was reduced in a highaltitude group compared with those in the normal altitude group; moreover, these high altitude subjects had longer response time and lower response accuracy, they believe that this may be caused by the long-term hypoxia concentration and low pressure of high altitude people, and the function and development of the brain in the hypoxia environment will be affected, which will lead to the decline in cognitive ability. ${ }^{33}$ In this instance, we can explain the abnormality of the basal ganglia network of asthmatic patients, because asthmatics are characterized by blocked airflow and bronchospasm, and severe asthma attacks can lead to hypoxia. ${ }^{34}$ Besides, many studies have shown that the insula is an important brain region involved in the neuropathology of asthma, for example, according to the research of Rosenkranz et al, patients with stress-related diseases such as asthma, regions of the brain such as the insula may lead to inflammatory responses by affecting disease-specific emotions and incoming physiological signals. ${ }^{13}$ Also, Zhang et al found that the FC value of the insular brain area of asthmatic patients decreased compared with that of normal patients, but after group cognitive behavior therapy treatment, the FC value of the insular subregion increased significantly. ${ }^{35}$ Additionally, the insula receives afferent information about the cord cortex of the thalamus, carries information related to respiration, and is closely related to the nerve center that processes emotions. ${ }^{36}$ Moreover, the insula is involved in the effective assessment of sensory stimuli, regulation of homeostasis responses, and visceral perception. Moreover, the degree of insula activation can predict individual differences in the evaluation of stimulus intensity and visceral consciousness. All of these studies indicate that emotional stimulation and insular area are closely related to asthma. In brief, we found a decrease in VMHC in basal ganglia, thalamus and the insular lobes, which may be used as a clinical indicator for evaluating BA.

Many pieces of evidence showed that asthma was a serious respiratory disease that can affect the nervous system, ${ }^{7,37}$ furthermore, there has been a functional MRI study with degree centrality (DC) that has confirmed that the sensorimotor network and visual network of asthmatic patients are abnormal. ${ }^{38}$ However, relevant studies on VMHC methods are still vacant. Brodmann Area (BA) $17 / 18 / 19$ is the visual cortex, these brain regions as the main area of the visual network, its VMHC value is altered in asthmatic patients, the abnormal change in the visual brain network may imply that asthma can affect the spontaneous changes of the central nervous system. These important brain regions of the visual network play an important role in the body's visual function. Calcarine (BA19) as the visual contact area, which is responsible for visual processing, and forms visual joint cortex with BA18, and a rs-fMRI study on asthma confirmed that the FC value of BA19 on the left side of asthma patients was significantly lower than that of the normal control group. ${ }^{35}$ Besides, cuneus (BA17) mainly belongs to the primary visual center (V1), as the core of visual pathways, ${ }^{39}$ it is responsible for the preliminary processing of visual information, receiving information about the lateral geniculate body, and transmitting the information to other visual areas, ${ }^{40}$ and when the body is not paying attention, visual information will be difficult to transmit from V1 to other regions. ${ }^{41}$ Also, the lingual gyrus (BA18) from the secondary visual cortex (V2) is the visual contact area, which is mainly responsible for visual processing. Moreover, it receives the pre feedback connection between V1 and plays an important role in object shape vision and stereo vision. ${ }^{42,43}$ Thus, we demonstrated that the neural activity of the visual network between the two hemispheres of asthmatic patients changed synchronously; moreover, this change of visual brain network in BA patients may lead to abnormal visual function.

PreCG (BA4/6, M1) and PostCG (BA3, S1) belong to the motor and sensory centers, respectively, they are also sensorimotor areas. ${ }^{44}$ In addition, the PostCG is the core component of the sensorimotor network, ${ }^{45}$ where the motor center mainly controls the movement behavior of the body, while the sensory center has the basic somatosensory function, which is responsible for encoding touch and pain, ${ }^{46}$ also, receiving the sensory input from the limbs. Furthermore, Liu et al found that the sensorimotor network of asthmatic patients would change, and this change was related to the altered respiratory amplitude, in addition, Li et al used MRI to contrast the brain structure of asthma patients and normal controls, found that patients with asthma sensorimotor network is abnormal, they through the network abnormal airway obstruction and the degree of correlation analysis reflect the brain damage associated with asthma caused by respiratory damage, they believe that brain network potential parameters can be used as an understanding of the pathophysiology of asthma. ${ }^{38}$ Also, Zhang et al found the M1/S1 brain area 
abnormality in asthmatic patients, and the $\mathrm{FC}$ value of these two brain regions was lower in asthmatic patients than that of the normal control group. ${ }^{35}$ Therefore, we hypothesized that sensorimotor function might change in BA patients, and this change was associated with abnormalities in key brain areas of the sensorimotor network.

Our further seed-based $\mathrm{rsFC}$ analyses revealed that $\mathrm{FC}$ values of these brain regions (B- PreCG/PostCG, B-Cuneus/Calcarine/Lingual Gyrus, and R-Basal ganglia/ Thalamus) were lower in BA, furthermore, the VMHC values corresponding to these brain regions in $\mathrm{BA}$ are also lower than those in normal subjects, which suggests that there are abnormal changes in resting-state functional connectivity between these brain regions in asthmatic patients and corresponding ataxia between cerebral hemispheres. Moreover, the inferior parietal lobule (ILP) is involved in the control of body perception, motor orientation, memory retrieval, language understanding, digital processing, and social cognition, this brain area is one of the main brain areas of the frontoparietal network, which plays an important role in attention and executive ability. ${ }^{47-49}$ Kuo et a found that the FC value of the ILP brain region in untreated heroin addicts was significantly lower than that in normal patients, and they speculated that the IPL brain region might be a neural target for treatment and intervention. ${ }^{50}$ Besides, an MRI study on asthma found that the degree centrality value of asthmatic patients in the IPL brain area was lower than that of normal controls, this study suggested that the IPL brain region can be used as one of the clinical indicators for the diagnosis of asthma. ${ }^{15}$ The supramarginal gyrus is involved in verbal divergent thinking, and this brain region is activated during divergent thinking tasks. ${ }^{51}$ The cingulate gyrus belongs to the limbic system, which is mainly involved in emotion formation, processing, learning, and memory, ${ }^{52}$ the interaction between the cingulate cortex and other brain networks may be very important to the body's consciousness. ${ }^{53}$ Furthermore, the caudate nucleus plays an important role in cognitive function, ${ }^{54}$ Zhang et al not only found the abnormality of FC value in asthma patients but also found the change of FC values in caudate and cingulate brain regions, ${ }^{35}$ which were similar to our findings in BA patients. Hence, combined with our rsFC results, we speculate that asthma may involve changes in functional connectivity of multiple brain regions, which are closely related to the body's emotional, motor, cognitive and other functions, and this may mean that asthma patients are dysfunctional in the above abilities.
However, our study did not collect the duration of BA and explore whether there is a correlation between $\mathrm{VMHC}$ values of brain regions changed by $\mathrm{BA}$, which is the deficiency of this study, and we will improve this point in the following work. Besides, our subjects are mainly middle-aged patients. We did not compare the differences of BA patients in different age groups, which is also the direction of our improvement in the future. In addition, although we have found the change of interhemispheric connectivity in patients with BA, we have not explored the specific mechanism. In the future, it will be our direction to study the specific mechanism of brain function and structural changes in patients with asthma.

\section{Conclusion}

In conclusion, VMHC and rsFC based on seed points were used to compare the resting-state functional connectivity between middle-aged BA individuals and healthy controls. We found that the functional connectivity between the hemispheres of bilateral basal ganglia, thalamus, insula, cuneus, calcarine, lingual gyrus, precentral and postcentral gyrus was abnormal in asthmatic patients, these results may help to understand the intrinsic neural mechanism of $\mathrm{BA}$, and provide some new reference to the formulation of treatment plans for asthma patients.

\section{Data Sharing Statement}

MRI data used to support the results of this study are available on request from the corresponding authors.

\section{Ethical Statements}

All research methods were approved by the Ethics Committee of People's Hospital affiliated Nanchang University. Informed consent was obtained from all participants before MRI scanning, and the research methods complied with the principles of the Declaration of Helsinki.

\section{Acknowledgments}

Thank you very much to Dr. Xiaorong Wu and Dr. Jun Wang for their guidance on the content of this article. Thanks to Dr. Xin HUANG for his charts and diagrams of the typesetting of pictures and texts. Thanks to Jie Rao for proofreading and modification of the article language. Thanks to $\mathrm{Na} \mathrm{Wu}$, Ling Shi, Hui Huang, Siyu Li, Xiaolin Chen, Shuiqin Huang, Peipei Zhong for their responsibility to clinical trials and data processing. Thank you for the support of NSFC, etc. Yajun Wu and Jie Rao are co-first authors for this study. 


\section{Author Contributions}

All authors made a significant contribution to the work reported, whether that is in the conception, study design, execution, acquisition of data, analysis, and interpretation, or in all these areas; took part in drafting, revising, or critically reviewing the article; gave final approval of the version to be published; have agreed on the journal to which the article has been submitted; and agree to be accountable for all aspects of the work.

\section{Funding}

National Natural Science Foundation of China (No.82160207, 81760179, 81360151); Health Development Planning Commission Science Foundation of Jiangxi Province (No. 20185118); Key research plan of Jiangxi Provincial Science and Technology Department (No.20192BBG70042); Key projects of Jiangxi Youth Science Fund (No.20202ACBL216008). National Natural Science Foundation of China (82160006); the Jiangxi Provincial Cultivation Program for Academic and Technical Leaders of Major Subjects (20172BCB22025); the Jiangxi Provincial Natural Science Fund Project (20202BAB206003).

\section{Disclosure}

The authors declare that the research was conducted in the absence of any commercial or financial relationships that could be construed as a potential conflict of interest.

\section{References}

1. Pawankar R. Allergic diseases and asthma: a global public health concern and a call to action. World Allergy Organ J. 2014;7(1):12. doi:10.1186/1939-4551-7-12

2. Lin J, Wang W, Chen P, et al. Prevalence and risk factors of asthma in mainland China: the CARE study. Respir Med. 2018;137:48-54. doi:10.1016/j.rmed.2018.02.010

3. Antó JM, Sunyer J, Basagaña X, et al. Risk factors of new-onset asthma in adults: a population-based international cohort study. Allergy. 2010;65(8):1021-1030. doi:10.1111/j.1398-9995.2009.02 301.x

4. Keskin O, Farzan N, Birben E, et al. Genetic associations of the response to inhaled corticosteroids in asthma: a systematic review. Clin Transl Allergy. 2019;9:2. doi:10.1186/s13601-018-0239-2. eCollection

5. Radon K, Büsching K, Heinrich J, et al. Passive smoking exposure: a risk factor for chronic bronchitis and asthma in adults? Chest. 2002;122(3):1086-1090. doi:10.1378/chest.122.3.1086

6. Koinis-Mitchell D, Kopel SJ, Seifer R, et al. Asthma-related lung function, sleep quality, and sleep duration in urban children. Sleep Health. 2017;3(3):148-156. doi:10.1016/j.sleh.2017.03.008

7. Rosenkranz MA, Davidson RJ. Affective neural circuitry and mind-body influences in asthma. Neuroimage. 2009;47(3):972-980. doi:10.1016/j.neuroimage.2009.05.042
8. Lomper K, Chudiak A, Uchmanowicz I, et al. Effects of depression and anxiety on asthma-related quality of life. Pneumonol Alergol Pol. 2016;84(4):212-221. doi:10.5603/PiAP.2016.0026

9. Choi S, Kim SH, Lee JS. Association between depression and asthma in Korean adults. Allergy Asthma Proc. 2017;38(3):37-46. doi:10.2500/aap.2017.38.4051

10. Caldera-Alvarado G, Khan DA, Defina LF, et al. Relationship between asthma and cognition: the Cooper Center Longitudinal Study. Allergy. 2013;68(4):545-548. doi:10.1111/all.12125

11. Xiong X, Zhu H, Wang T, Ji Y. Altered intrinsic regional brain activity in female asthmatics with or without depressive symptoms: a resting-state functional magnetic resonance imaging study. J Asthma. 2016;53(9):922-929. doi:10.3109/02770903.2016.1161050

12. Kline JN, Rose RM. Central nervous system influences in asthma. $A d v$ Exp Med Biol. 2014;795:309-319. doi:10.1007/978-1-4614-8603-9_19

13. Rosenkranz MA, Busse WW, Johnstone T, et al. Neural circuitry underlying the interaction between emotion and asthma symptom exacerbation. Proc Natl Acad Sci USA. 2005;102(37):13319-13324. doi:10.1073/pnas.0504365102

14. Zhang Y, Yang Y, Bian R, et al. Abnormal functional connectivity of ventral anterior insula in asthmatic patients with depression. Neural Plast. 2017;2017:7838035. doi:10.1155/2017/7838035

15. Li QG, Zhou FQ, Huang X, et al. Alterations of resting-state functional network centrality in patients with asthma: evidence from a voxel-wise degree centrality analysis. Neuroreport. 2018;29 (14):1151-1156. doi:10.1097/WNR.0000000000001087

16. Carlson SM, Kim J, Khan DA, et al. Hippocampal volume in patients with asthma: results from the Dallas Heart Study. J Asthma. 2017;54 (1):9-16. doi:10.1080/02770903.2016.1186174

17. Von Leupoldt A, Brassen S, Baumann HJ, et al. Structural brain changes related to disease duration in patients with asthma. PLoS One. 2011;6(8):e23739. doi:10.1371/journal.pone.0023739

18. Bian R, Zhang Y, Yang Y, et al. White matter integrity disruptions correlate with cognitive impairments in asthma. J Magn Reson Imaging. 2018;21. doi:10.1002/jmri.25946

19. Terada K, Usui N, Umeoka S, et al. Interhemispheric connection of motor areas in humans. J Clin Neurophysiol. 2008;25(6):351-356. doi:10.1097/WNP.0b013e31818f4fec

20. Bernasconi F, Grivel J, Murray MM, et al. Interhemispheric coupling between the posterior sylvian regions impacts successful auditory temporal order judgment. Neuropsychologia. 2010;48 (9):2579-2585. doi:10.1016/j.neuropsychologia.2010.05.004

21. Mima T, Oluwatimilehin T, Hiraoka T, et al. Transient interhemispheric neuronal synchrony correlates with object recognition. $J$ Neurosci. 2001;21(11):3942-3948. doi:10.1523/JNEUROSCI.21-11-03942.2001

22. Zuo XN, Kelly C, Di Martino A, et al. Growing together and growing apart: regional and sex differences in the lifespan developmental trajectories of functional homotopy. $J$ Neurosci. 2010;30 (45):15034-15043. doi:10.1523/JNEUROSCI.2612-10.2010

23. Liu YT, Zhang HX, Li HJ, et al. Aberrant interhemispheric connectivity in obstructive sleep apnea-hypopnea syndrome. Front Neurol. 2018;9:314. doi:10.3389/fneur.2018.00314

24. Zhou F, Zhao Y, Huang M, et al. Disrupted interhemispheric functional connectivity in chronic insomnia disorder: a resting-state fMRI study. Neuropsychiatr Dis Treat. 2018;14:1229-1240. doi:10.2147/NDT. S162325

25. Yan CG, Wang XD, Zuo XN, et al. DPABI: data processing \& analysis for (Resting-State) brain imaging. Neuroinformatics. 2016;14(3):339-351. doi:10.1007/s12021-016-9299-4

26. Van Dijk KR, Sabuncu MR, Buckner RL. The influence of head motion on intrinsic functional connectivity MRI. Neuroimage. 2012;59(1):431-438. doi:10.1016/j.neuroimage.2011.07.044

27. Goto M, Abe O, Aoki S, et al. Diffeomorphic anatomical registration through exponentiated Lie Algebra provides reduced effect of scanner for cortex volumetry with atlas-based method in healthy subjects. Neuroradiology. 2013;55(7):869-875. doi:10.1007/s00234-013-1193-2 
28. Song XW, Dong ZY, Long XY, et al. REST: a toolkit for resting-state functional magnetic resonance imaging data processing. PLoS One. 2011;6(9):e25031. doi:10.1371/journal.pone.0025031

29. Guo W, Liu F, Dai Y, et al. Decreased interhemispheric resting-state functional connectivity in first-episode, drugnaive major depressive disorder. Prog Neuropsychopharmacol Biol Psychiatry. 2013;41:24-29. doi:10.1016/j.pnpbp.2012.11.003

30. Ritz T, Kroll JL, Patel SV, et al. Central nervous system signatures of affect in asthma: associations with emotion-induced bronchoconstriction, airway inflammation, and asthma control. $J$ Appl Physiol. 2019;126(6):1725-1736. doi:10.1152/japplphysiol.01018.2018

31. Kwon DH, Paek SH, Kim YB, et al. In vivo 3D reconstruction of the human pallidothalamic and nigrothalamic pathways with super-resolution 7T MR track density imaging and fiber tractography. Front Neuroanat. 2021;15:739576. doi:10.3389/ fnana.2021.739576

32. Mantel T, Meindl T, Li Y, et al. Network-specific resting-state connectivity changes in the premotor-parietal axis in writer's cramp. Neuroimage Clin. 2017;17:137-144. doi:10.1016/j.nicl.2017.10.001

33. Xiaodan Y, Jiaxing Z, Qiyong G, et al. Prolonged high-altitude residence impacts verbal working memory: an fMRI study. Exp Brain Res. 2011;208(3):437-445. doi:10.1007/s00221-010-2494-x

34. Eckert DJ, Catcheside PG, Smith JH, et al. Hypoxia suppresses symptom perception in asthma. Am $J$ Respir Crit Care Med. 2004;169(11):1224-1230. doi:10.1164/rccm.200305-630OC

35. Yuqun Z, Yang Y, Rongrong B, et al. Group cognitive behavior therapy reversed insula subregions functional connectivity in asthmatic patients. Front Aging Neurosci. 2017;9:105. doi:10.3389/ fnagi.2017.00105

36. Borsook D, Veggeberg, Erpelding N, et al. The insula: a "hub of activity" in migraine. Neuroscientist. 2016;22(6):632-652. doi:10.1177/1073858415601369

37. Parker J, Wolansky LJ, Khatry D, et al. Brain magnetic resonance imaging in adults with asthma. Contemp Clin Trials. 2011;32 (1):86-89. doi:10.1016/j.cct.2010.09.006

38. Li S, Lv P, He M, et al. Cerebral regional and network characteristics in asthma patients: a resting-state fMRI study. Front Med. 2020;14 (6):792-801. doi:10.1007/s11684-020-0745-1

39. Callaway EM. Neural substrates within primary visual cortex for interactions between parallel visual pathways. Prog Brain Res. 2005;149:59-64. doi:10.1016/S0079-6123(05)49005-6

40. Atapour N, Worthy KH, Lui LL, et al. Neuronal degeneration in the dorsal lateral geniculate nucleus following lesions of primary visual cortex: comparison of young adult and geriatric marmoset monkeys. Brain Struct Funct. 2017;222(7):3283-3293. doi:10.1007/s00429-017-1404-4

41. Zhao PL. A new framework for understanding vision from the perspective of the primary visual cortex. Curr Opin Neurobiol. 2019;58:1-10. doi:10.1016/j.conb.2019.06.001
42. Hegdé J, Van Essen DC. Strategies of shape representation in macaque visual area V2. Vis Neurosci. 2003;20(3):313-328. doi:10.1017/ s0952523803203102

43. Von der Heydt R, Zhou H, Friedman HS. Representation of stereoscopic edges in monkey visual cortex. Vision Res. 2000;40 (15):1955-1967. doi:10.1016/s0042-6989()00044-400

44. Liu Y, Qin W, Li R, et al. Investigation on the neural mechanism of hypnosis-based respiratory control using functional MRI. Contrast Media Mol Imaging. 2018;2018:8182542. doi:10.1155/2018/8182542

45. Rocca MA, Agosta F, Mezzapesa DM, et al. A functional MRI study of movement-associated cortical changes in patients with Devic's neuromyelitis optica. Neuroimage. 2004;21(3):1061-1068. doi:10.1016/j.neuroimage.2003.10.013

46. Gazzola V, Spezio ML, Etzel JA, et al. Primary somatosensory cortex discriminates affective significance in social touch. Proc Natl Acad Sci USA. 2012;109(25):E1657-66. doi:10.1073/pnas.1113211109

47. Caspers S, Schleicher A, Trams MB, et al. Organization of the human inferior parietal lobule based on receptor architectonics. Cerebral Cortex. 2013;23(3):615-628. doi:10.1093/cercor/bhs048

48. Chen AC, Oathes DJ, Chang C, et al. Causal interactions between fronto-parietal central executive and defaultmode networks in humans. Proc Natl Acad Sci USA. 2013;110(49):19944-19949. doi:10.1073/pnas. 1311772110

49. Markett S, Reuter M, Montag C, et al. Assessing the function of the fronto-parietal attention network: insights from resting-state fMRI and the attentional network test. Hum Brain Mapp. 2014;35 (4):1700-1709. doi:10.1002/hbm.22285

50. Kuo LW, Lin PS, Lin SY, et al. Functional correlates of resting- state connectivity in the default mode network of heroin users on methadone treatment and medication-free therapeutic community program. Front Psychiatry. 2019;10:381. doi:10.3389/fpsyt.2019.00381

51. Cousijn J, Zanolie K, Munsters RJ, et al. The relation between resting state connectivity and creativity in adolescents before and after training. PLoS One. 2014;9(9):e105780. doi:10.1371/journal.pone.0105780

52. Kozlovskiy SA, Vartanov AV, Nikonova, et al., The cingulate cortex and human memory processes. Psychol Russia. 2012;5:231-243.

53. Leech R, Sharp DJ. The role of the posterior cingulate cortex in cognition and disease. Brain. 2014;137(Pt 1):12-32. doi:10.1093/ brain/awt162

54. Jaspers E, Balsters JH, Fard K, et al. Corticostriatal connectivity fingerprints: probability maps based on resting-state functional connectivity. Hum Brain Mapp. 2017;38(3):1478-1491. doi:10.1002/hbm.23466
International Journal of General Medicine

\section{Publish your work in this journal}

The International Journal of General Medicine is an international, peer-reviewed open-access journal that focuses on general and internal medicine, pathogenesis, epidemiology, diagnosis, monitoring and treatment protocols. The journal is characterized by the rapid reporting of reviews, original research and clinical studies across all disease areas. The manuscript management system is completely online and includes a very quick and fair peer-review system, which is all easy to use. Visit http://www.dovepress.com/ testimonials.php to read real quotes from published authors. 